\title{
Transport metabolons with carbonic anhydrases
}

\author{
Joachim W. Deitmer ${ }^{1 *}$ and Holger M. Becker ${ }^{2}$ \\ ${ }^{1}$ General Zoology, University of Kaiserslautern, Kaiserslautern, Germany \\ 2 Zoology/Membrane Transport, FB Biologie, University of Kaiserslautern, Kaiserslautern, Germany \\ *Correspondence: deitmer@biologie.uni-kl.de \\ Edited by: \\ Ebbe Boedtkjer, Aarhus University, Denmark \\ Reviewed by: \\ Ira Kurtz, University of California, Los Angeles, USA
}

Keywords: acid/base, $\mathrm{pH}$, proton, bicarbonate, lactate

\section{THE CONCEPT OF THE TRANSPORT METABOLON}

A metabolon has been defined as a "temporary, structural-functional, supramolecular complex of sequential metabolic enzymes and cellular structural elements, in which metabolites are passed from one active site to another without complete equilibration with the bulk cellular fluids" (Srere, 1985, 1987). This substrate channeling should decrease transit time of intermediates, prevent loss of intermediates by diffusion, protect labile intermediates from solvent, and prevent entrance of intermediates into competing metabolic pathways (Miles et al., 1999). Such metabolons have been found in many metabolic pathways like glycolysis and tricarboxylic acid cycle, as well as in the biosynthesis of DNA, RNA, and proteins (Srere, 1987).

Some of the major acid/base-coupled membrane transporters, such as the $\mathrm{Cl}^{-} / \mathrm{HCO}_{3}^{-}$exchanger $\mathrm{AE} 1$ (anion exchanger 1; Band3), the $\mathrm{Na}^{+} / \mathrm{H}^{+}$ exchanger NHE1, the $\mathrm{Na}^{+} / \mathrm{HCO}_{3}^{-}$ cotransporter NBCe1, and various monocarboxylate transporters (MCTs) have been demonstrated to physically and/or functionally interact with isoforms of carbonic anhydrase (CA) to form a "transport metabolon." This functional interaction includes an increase in transport activity, and therefore may enhance both the rate and the capacity of ion/metabolite transport in tissues where these membrane transporters and enzymes are co-localized.

\section{INTERACTIONS REOUIRING CATALYTIC CA ACTIVITY}

First evidence for a transport metabolon, formed between CA and an acid/base transporter, has been presented in 1993 by Kifor et al. (1993) for CAII and AE1. CAII could be co-immunoprecipitated with AE1 when antiserum against the $\mathrm{N}$ terminal of AE1 was used, while immunoprecipitation of AE1 with serum directed against the C-terminal of the transporter failed to co-immunoprecipitate CAII (Vince and Reithmeier, 1998), suggesting that CAII physically binds to the C-terminal tail of AE1. These data were confirmed by affinity blotting and a solid phase binding assay with CAII and a GST fusion protein of the AE1 Cterminal, which showed saturable binding of CAII with a $\mathrm{K}_{1 / 2}$ of $20 \mathrm{nM}$ (Vince and Reithmeier, 1998). Single site mutations identified the acidic cluster $\mathrm{L}^{886} \mathrm{DADD}$ in the C-terminal tail of AE1 as binding site for CAII with at least 2 out of the 3 acidic amino acids being crucial for binding (Vince and Reithmeier, 2000; Vince et al., 2000). This suggests a hydrophobic amino acid followed by at least two acidic amino acids as the CAII binding motif. The amino acid cluster $\mathrm{L}^{886} \mathrm{DADD}$ in the C-terminal of AE1 is located close to the last transmembrane domain. Therefore, binding of CAII to this cluster would position the enzyme close to the transporter pore of AE1 near the inner cell surface. This location may ideally position CAII to hydrate incoming $\mathrm{CO}_{2}$ and directly supply the AE1 transporter with a localized substrate pool (Vince and Reithmeier, 2000). Indeed, inhibition of CAII catalytic activity decreased transport activity of AE1 heterologously expressed in HEK293 cells by up to $60 \%$ (Sterling et al., 2001).

Reports on a functional interaction between NBCs and carbonic anhydrase go back to the finding that application of the CA inhibitor acetazolamide inhibits transport of bicarbonate across the basolateral cell membrane in renal proximal tubule of rabbits (Burg and Green, 1977; Sasaki and Marumo, 1989; Seki and Frömter, 1992). First evidence for a direct interaction between NBCe1 and CAII was presented by Gross et al. (2002) using isothermal titration calorimetry. CAII was found to bind to the C-terminal peptide of kNBCel (amino acids 915-1035) with a $\mathrm{K}_{\mathrm{D}}$ of $160 \pm 10 \mathrm{nM}$. In analogy to the acidic CAII-binding cluster $\mathrm{D}^{887} \mathrm{ADD}$ found in AE1 (Vince and Reithmeier, 2000), the authors suggested the cluster $\mathrm{D}^{986} \mathrm{NDD}$ within the $\mathrm{C}$-terminal of NBCel as the putative CAII binding site. Functional interaction between NBCel and CAII was further investigated by heterologous protein expression in Xenopus oocytes (Becker and Deitmer, 2007): Both injection and coexpression of CAII increased NBCe1-mediated membrane current and membrane conductance during application of $\mathrm{CO}_{2} / \mathrm{HCO}_{3}^{-}$-buffered solution in an ethoxzolamide-sensitive manner. Measurements of intracellular $\mathrm{Na}^{+}$concentrations with ion-selective microelectrodes showed an increase in the rate of NBCel-mediated $\mathrm{Na}^{+}$-flux by two- to four-fold when CAII was injected or co-expressed. CAII-mediated increase in NBCel activity as determined by changes in membrane current and slope conductance was dependent on the CAII concentration with a halfmaximum enhancement in NBCel activity at 20-30 ng CAII/oocyte (Becker and Deitmer, 2007).

Physical and functional interaction with extracellular CAIV could also be demonstrated for the $\mathrm{Na}^{+} / \mathrm{HCO}_{3}^{-}$ cotransporter NBCe1 (Alvarez et al., 2003): Transport activity of NBCe1 was determined by fluorometric $\mathrm{pH}$ 
measurements in NBCe1-transfected HEK293 cells subjected to acid loads. Co-transfection of NBCe1 with CAIV significantly increased the rate of NBCe1mediated $\mathrm{pH}_{\mathrm{i}}$ recovery. In contrast, CAIV did not increase activity of the NBCe1mutant $\mathrm{G}^{767} \mathrm{~T}$ (positioned in the 4th extracellular loop). In line with the physiological findings, pull-down assays demonstrated physical binding between CAIV and a GST fusion protein of the NBCe1's 4th extracellular loop, but neither to a GST fusion protein of the 4th extracellular loop in which $\mathrm{G}^{767}$ was mutated to T, nor to a GST fusion protein of the transporter's 3rd extracellular loop. These data indicate that CAIV can bind to the 4th extracellular loop of NBCe1 [as it binds to the 4th extracellular loop of AE1 (Sterling et al., 2002)] to form the extracellular part of a CAIV-NBCe1 transport metabolon (Alvarez et al., 2003). A transport metabolon of NBCe1 and AE2 with CAIX has recently been suggested also for migrating MDCK cells (Svastova et al., 2012).

It should be noted, however, that the interactions between anion carriers and CAs as described above, have been disputed with respect to the binding and transport activity of the proteins involved (Lu et al., 2006; Piermarini et al., 2007; Yamada et al., 2011). The transport activity was evaluated only by either the current or by the slope conductance in two of these studies, - parameters which may vary in oocytes to a degree which make it difficult to isolate the component contributed by CA, which could be less than $20 \%$. On the other hand, CAII activity may improve substrate supply to bicarbonate transporters even without the requirement for a metabolon involving direct physical interaction, as also pointed out in a recent study on AE1 transport activity (AlSamir et al., 2013). Therefore, while there is growing support for a functional interaction between bicarbonate transporters and CA activity, the question whether this interaction requires direct binding of the proteins involved remains not finally settled at this point.

\section{INTERACTIONS INDEPENDENT OF CATALYTIC CA ACTIVITY}

Lactate, pyruvate, and ketone bodies are transported into and out of cells via monocarboxylate transporters (MCT, SLC16), of which 14 isoforms have been described. The first four of these 14 isoforms (MCT1-4) have been shown to transport monocarboxylates together with $\mathrm{H}^{+}$in a 1:1 stoichiometry (Carpenter and Halestrap, 1994; Bröer et al., 1998). All MCTs have a classical 12 transmembranehelix structure, with both the C- and Nterminal located intracellularly (Halestrap and Price, 1999). Trafficking, but also regulation of transport activity of MCT1-4 is mediated by the ancillary proteins basigin (CD147) or embigin (gp70), which bind to the transporter (Wilson et al., 2005).

First evidence that the non-catalytic interaction between MCT and CAII depends on a direct interaction between the two proteins was shown by injection of CAII that was bound to an antibody prior to the injection. In this experiment CAII was not able to enhance transport activity of MCT1 in Xenopus oocytes, suggesting a steric suppression of the interaction by the antibody (Becker et al., 2005). In the same study, truncation of the MCT1 C-terminal tail (MCT1-D56) led to loss of interaction between MCT1 and CAII in Xenopus oocytes.

By introduction of single site mutations in the C-terminal of MCT1 and subsequent expression of these mutants in CAII-injected Xenopus oocytes, the two glutamate residues $\mathrm{E}^{489}$ and $\mathrm{E}^{491}$ flanking the acidic cluster $\mathrm{E}^{489} \mathrm{EE}$ within the MCT1 C-terminal tail could be identified to be crucial for the functional interaction with CAII (Stridh et al., 2012). Direct binding between CAII and the MCT1 C-terminal tail was shown by co-immunoprecipitation when the acidic cluster $\mathrm{E}^{489} \mathrm{EE}$ was intact, while mutation of $\mathrm{E}^{489}$ and/or $\mathrm{E}^{491}$ suppressed the binding between MCT1-CT and CAII. This suggests that cytosolic CAII can bind to the C-terminal tail of MCT1, which presumably positions the enzyme close enough to the pore of the transporter for efficient $\mathrm{H}^{+}$ shuttling.

It has been demonstrated that the enhancing effect of CAII on $\mathrm{H}^{+}$/lactate influx via MCT1 and MCT4 increased with increasing extracellular proton concentration, but decreased with extracellular lactate concentration (Becker and Deitmer, 2008; Almquist et al., 2010; Becker et al., 2010). This gave rise to the hypothesis that CAII-induced augmentation of MCT transport activity is linked to the $\mathrm{H}^{+}$gradient across the cell membrane. Mathematical modeling of the transport mechanism suggested that CAII increases the rate constant for the binding and the release of $\mathrm{H}^{+}$at the transporter by providing additional $\mathrm{H}^{+}$binding sites, and thereby speeds up lactate/proton cotransport (Almquist et al., 2010).

CAII facilitates $\mathrm{H}^{+}$transfer between the zinc-bound water and the solvent surrounding the enzyme by the side chain of $\mathrm{H} 64$, which shuttles $\mathrm{H}^{+}$between the bulk solvent and a network of well-ordered hydrogen-bonded water molecules in the enzyme's active-site cavity (Fisher et al., 2007). Co-expression of the mutant CAII$\mathrm{H} 64 \mathrm{~A}$, lacking the intramolecular $\mathrm{H}^{+}$ shuttle, with MCT1 or MCT4 in Xenopus oocytes resulted in no increase in transport activity (Becker et al., 2011). This led to the notion that CAII may provide or subtract protons to or from the transporter, respectively, via its intramolecular $\mathrm{H}^{+}$ shuttle. Injection of 4-methylimidazole (4MI), which acts as a $\mathrm{H}^{+}$donor/acceptor, indeed restored the ability of the CAIIH64A mutant to enhance transport activity of MCT1/4 (Becker et al., 2011). It is hypothesized that 4-MI binds at various positions within the active site cavity of CAII, which would then rescue proton shuttling in the enzyme (Duda et al., 2001).

While injection of CAII does not increase transport activity of MCT2, heterologously expressed in Xenopus oocytes, co-expression of the extracellular isoform CAIV led to a doubling in the rate of lactate-induced acidification (Klier et al., 2011). As already observed for MCT $1 / 4$ and CAII, the interaction between MCT2 and CAIV persisted in the nominal absence of $\mathrm{CO}_{2} / \mathrm{HCO}_{3}^{-}$and was insensitive to inhibition of the enzyme's catalytic activity. The non-catalytic nature of the interaction was confirmed by coexpression of MCT2 with the catalytically inactive mutant CAIV-V165Y, which increased MCT2 activity as did CAIV-WT. Interestingly, removing the intramolecular $\mathrm{H}^{+}$-shuttle (CAIV-H88A), analogous to the proton shuttle in CAII, which had been shown to be crucial for the interaction of CAII with MCT1/4, led to a significantly smaller increase in MCT2 
activity than did CAIV-WT, but did not fully abolish the interaction with MCT2 (Klier et al., 2011). It has been suggested that this residual enhancement could be due to a second, not yet identified, proton shuttle in CAIV (Hurt et al., 1997; Klier et al., 2011). Furthermore, CAIV could only increase activity of MCT2 when the transporter was co-expressed with its trafficking protein embigin (gp70). From this it was concluded that the interaction between MCT2 and CAIV may not depend on a direct binding between MCT2 and CAIV, as suggested for MCT1/4 and CAII, but may be mediated by binding of CAIV to embigin, which could act as a mediator for CAIV-induced transport enhancement of MCT2 (Klier et al., 2011). As both N- and C-terminal tails of MCT2 are located intracellularly, and the transporters' short extracellular loops might not provide enough space for binding of CAIV, the two extracellular globular domains of embigin may well provide a binding site for CAIV.

\section{CONCLUSIONS AND PERSPECTIVES}

Transport metabolons between carbonic anhydrases and acid/base-coupled membrane transporters display a great versatility. Not only that most of the $\mathrm{H}^{+}$and $\mathrm{HCO}_{3}^{-}$carrying transporters interact with one or several isoforms of carbonic anhydrase, the mode of interaction also shows some unexpected variability. Membrane transporters may interact with intracellular and extracellular carbonic anhydrases, and this interaction may be direct including physical binding, or may possibly be mediated by chaperones associated with the transport proteins. Most of the interactions require the catalytic activity of the carbonic anhydrases, and functional interaction may be attributable mainly to the faster conversion of $\mathrm{CO}_{2}, \mathrm{HCO}_{3}^{-}$, and $\mathrm{H}^{+}$ by CAs, while co-localization and perhaps physical attachment between acid/basecoupled transporters and CAs may meet the structural and spatial requirements of this process. The isoform-specific interplay between all monocarboxylate transporters and carbonic anhydrase isoforms so far tested, is independent of the anhydrase enzymatic activity. Moreover, similar binding domains have been identified in various MCT and CA isoforms. Future studies on the interaction between transporters and carbonic anhydrases, should help to unravel the molecular mechanisms of this functional metabolon system in more detail, from which we may learn more about transport metabolons in general.

\section{ACKNOWLEDGMENTS}

The authors' own contributions to the field have been supported by the Deutsche Forschungsgemeinschaft, the Stiftung Rheinland-Pfalz für Innovation, and the Research Initiative in Membrane Biology at the University of Kaiserslautern.

\section{REFERENCES}

Almquist, J., Lang, P., Prätzel-Wolters, D., Deitmer, J. W., Jirstrand, M., and Becker, H. M. (2010). A kinetic model of the monocarboxylate transporter MCT1 and its interaction with carbonic anhydrase II. J. Comput. Sci. Syst. Biol. 3, 107-116. doi: 10.4172/jcsb.1000066

Al-Samir, S., Papadopoulos, S., Scheibe, R. J., Meißner, J. D. Cartron, J. P., Sly, W. S., et al. (2013). Activity and distribution of intracellular carbonic anhydrase II and their effects on the transport activity of anion exchanger AE1/SLC4A1. J. Physiol. doi: 10.1113/jphysiol.2013.251181. [Epub ahead of print].

Alvarez, B. V., Loiselle, F. B., Supuran, C. T., Schwartz, G. J., and Casey, J. R. (2003). Direct extracellular interaction between carbonic anhydrase IV and the human NBC1 sodium/bicarbonate cotransporter. Biochemistry 42, 12321-12329. doi: 10.1021/bi0353124

Becker, H. M., and Deitmer, J. W. (2007). Carbonic anhydrase II increases the activity of the human electrogenic $\mathrm{Na}^{+} / \mathrm{HCO}_{3}^{-}$cotransporter. J. Biol. Chem. 282, 13508-13521. doi: 10.1074/jbc.M700 066200

Becker, H. M., and Deitmer, J. W. (2008). Nonenzymatic proton handling by carbonic anhydrase II during $\mathrm{H}^{+}$-lactate cotransport via monocarboxylate transporter 1. J. Biol. Chem. 283, 21655-21667. doi: 10.1074/jbc.M802134200

Becker, H. M., Hirnet, D., Fecher-Trost, C. Sültemeyer, D., and Deitmer, J. W. (2005). Transport activity of MCT1 expressed in Xenopus oocytes is increased by interaction with carbonic anhydrase. J. Biol. Chem. 280, 39882-39889. doi: 10.1074/jbc.M503081200

Becker, H. M., Klier, M., and Deitmer, J. W. (2010). Nonenzymatic augmentation of lactate transport via monocarboxylate transporter isoform 4 by carbonic anhydrase II. J. Membr. Biol. 234, 125-135. doi: 10.1007/s00232-010-9240-y

Becker, H. M., Klier, M., Schüler, C., McKenna, R., and Deitmer, J. W. (2011). Intramolecular proton shuttle supports not only catalytic but also noncatalytic function of carbonic anhydrase II. Proc. Natl. Acad. Sci. U.S.A. 108, 3071-3076. doi: 10.1073/pnas. 1014293108

Bröer, S., Schneider, H. P., Bröer, A., and Deitmer, J. W. (1998). Characterization of the monocarboxylate transporter 1 expressed in Xenopus laevis oocytes by changes in cytosolic pH. Biochem. J. 174, 167-174.

Burg, M., and Green, N. (1977). Bicarbonate transport by isolated perfused rabbit proximal convoluted tubules. Am. J. Physiol. 233, 307-314.

Carpenter, L., and Halestrap, A. P. (1994). The kinetics, substrate and inhibitor specificity of the lactate transporter of Ehrlich-Lettre tumour cells studied with the intracellular $\mathrm{pH}$ indicator BCECF. Biochem. J. 304, 751-760.

Duda, D., Tu, C., Qian, M., Laipis, P., AgbandjeMcKenna, M., Silverman, D. N., et al. (2001). Structural and kinetic analysis of the chemical rescue of the proton transfer function of carbonic anhydrase II. Biochemistry 40, 1741-1748. doi: 10.1021/bi002295z

Fisher, S. Z., Maupin, C. M., Budayova-Spano, M., Govindasamy, L., Tu, C., Agbandje-McKenna, M., et al. (2007). Atomic crystal and molecular dynamics simulation structures of human carbonic anhydrase II: insights into the proton transfer mechanism. Biochemistry 46, 2930-2937. doi: 10.1021/bi062066y

Gross, E., Pushkin, A., Abuladze, N., Fedotoff, O., and Kurtz, I. (2002). Regulation of the sodium bicarbonate cotransporter $\mathrm{kNBC1}$ function: role of Asp986, Asp988 and kNBC1-carbonic anhydrase II binding. J. Physiol. 544, 679-685. doi: 10.1113/jphysiol.2002.029777

Halestrap, A. P., and Price, N. T. (1999). The proton-linked monocarboxylate transporter (MCT) family: structure, function and regulation. Biochem. J. 343, 281-299. doi: 10.1042/0264-6021: 3430281

Hurt, J. D., Tu, C., Laipis, P. J., and Silverman, D. N. (1997). Catalytic properties of murine carbonic anhydrase IV. J. Biol. Chem. 272, 13512-13518. doi: 10.1074/jbc.272.21.13512

Kifor, G., Toon, M. R., Janoshazi, A., and Solomon, A. K. (1993). Interaction between red cell membrane band 3 and cytosolic carbonic anhydrase. J. Membr. Biol. 134, 169-179. doi: 10.1007/BF00 234498

Klier, M., Schüler, C., Halestrap, A. P., Sly, W. S., Deitmer, J. W., and Becker, H. M. (2011). Transport activity of the high-affinity monocarboxylate transporter MCT2 is enhanced by extracellular carbonic anhydrase IV but not by intracellular carbonic anhydrase II. J. Biol. Chem. 286, 27781-27791. doi: 10.1074/jbc. M111.255331

Lu, J., Daly, C. M., Parker, M. D., Gill, H. S., Piermarini, P. M., Pelletier, M. F., et al. (2006). Effect of human carbonic anhydrase II on the activity of the human electrogenic $\mathrm{Na} / \mathrm{HCO} 3$ cotransporter NBCe1-A in Xenopus oocytes. J. Biol. Chem. 281, 19241-19250. doi: 10.1074/jbc.M602181200

Miles, E. W., Rhee, S., and Davies, D. R. (1999). The molecular basis of substrate channeling. J. Biol. Chem. 274, 12193-12196. doi: 10.1074/jbc.274. 18.12193

Piermarini, P. M., Kim, E. Y., and Boron, W. F. (2007). Evidence against a direct interaction between intracellular carbonic anhydrase II and pure C-terminal domains of SLC4 bicarbonate transporters. J. Biol. Chem. 282, 1409-1421. doi: 10.1074/jbc.M608261200 
Sasaki, S., and Marumo, F. (1989). Effects of carbonic anhydrase inhibitors on basolateral base transport of rabbit proximal straight tubule. Am. J. Physiol. 257, 947-952.

Seki, G., and Frömter, E. (1992). Acetazolamide inhibition of basolateral base exit in rabbit renal proximal tubule S2 segment. Pflügers. Archiv. Eur. J. Physiol. 422, 60-65. doi: 10.1007/BF00381514

Srere, P. A. (1985). The metabolon. Trends. Biochem. Sci. 14, 313-314.

Srere, P. A. (1987). Complexes of sequential metabolic enzymes. Annu. Rev. Biochem. 56, 89-124. doi: 10.1146/annurev.bi.56.070187.000513

Sterling, D., Alvarez, B. V., and Casey, J. R. (2002). The extracellular component of a transport metabolon. Extracellular loop 4 of the human AE1 $\mathrm{Cl}^{-} / \mathrm{HCO}_{3}^{-}$exchanger binds carbonic anhydrase IV. J. Biol. Chem. 277, 25239-25246. doi: 10.1074/jbc.M202562200

Sterling, D., Reithmeier, R. A., and Casey, J. R. (2001). A transport metabolon. Functional interaction of carbonic anhydrase II and chloride/bicarbonate exchangers. J. Biol. Chem. 276, 47886-47894.

Stridh, M. H., Alt, M. D., Wittmann, S., Heidtmann, H., Aggarwal, M., Riederer, B., et al. (2012). Lactate flux in astrocytes is enhanced by a non-catalytic action of carbonic anhydrase II. J. Physiol. 590, 2333-2351. doi: 10.1113/jphysiol.2011.220152
Svastova, E., Witarski, W., Csaderova, L., Kosik, I., Skarkova, L., Hulikova, A., et al. (2012). Carbonic anhydrase IX interacts with bicarbonate transporters in lamellipodia and increases cell migration via its catalytic domain. J. Biol. Chem. 287, 3392-3402. doi: 10.1074/jbc.M111. 286062

Vince, J. W., Carlsson, U., and Reithmeier, R. A. (2000). Localization of the $\mathrm{Cl}^{-} / \mathrm{HCO}_{3}^{-}$anion exchanger binding site to the amino-terminal region of carbonic anhydrase II. Biochemistry 39, 13344-13349. doi: 10.1021/bi0015111

Vince, J. W., and Reithmeier, R. A. (1998). Carbonic anhydrase II binds to the carboxyl terminus of human band 3, the erythrocyte $\mathrm{Cl}^{-} / \mathrm{HCO}_{3}^{-}$ exchanger. J. Biol. Chem. 273, 28430-28437. doi: 10.1074/jbc.273.43.28430

Vince, J. W., and Reithmeier, R. A. (2000). Identification of the carbonic anhydrase II binding site in the $\mathrm{Cl}(-) / \mathrm{HCO}(3)(-)$ anion exchanger AE1. Biochemistry 39, 5527-5533. doi: 10.1021/bi9 $92564 \mathrm{p}$

Wilson, M. C., Meredith, D., Fox, J. E. M., Manoharan, C., Davies, A. J., and Halestrap, A. P. (2005). Basigin (CD147) is the target for organomercurial inhibition of monocarboxylate transporter isoforms 1 and 4: the ancillary protein for the insensitive MCT2 is EMBIGIN (gp70).
J. Biol. Chem. 280, 27213-27221. doi: 10.1074/jbc. M411950200

Yamada, H., Horita, S., Suzuki, M., Fujita, T., and Seki, G. (2011). Functional role of a putative carbonic anhydrase II-binding domain in the electrogenic $\mathrm{Na}^{+}-\mathrm{HCO}_{3}^{-}$cotransporter $\mathrm{NBCe} 1$ expressed in Xenopus oocytes. Channels 5, 1-4. doi: $10.4161 /$ chan.5.2.14341

Received: 26 August 2013; accepted: 24 September 2013; published online: 10 October 2013.

Citation: Deitmer JW and Becker HM (2013) Transport metabolons with carbonic anhydrases. Front. Physiol. 4:291. doi: 10.3389/fphys.2013.00291

This article was submitted to Membrane Physiology and Membrane Biophysics, a section of the journal Frontiers in Physiology.

Copyright (c) 2013 Deitmer and Becker. This is an open-access article distributed under the terms of the Creative Commons Attribution License (CC BY). The use, distribution or reproduction in other forums is permitted, provided the original author(s) or licensor are credited and that the original publication in this journal is cited, in accordance with accepted academic practice. No use, distribution or reproduction is permitted which does not comply with these terms. 